\title{
A comparative analysis of speech rate and perception in radio bulletins
}

\author{
EMMA RODERO
}

\begin{abstract}
Speech rate is one of the most important elements in a news presentation, especially on radio, a sound medium. Accordingly, this study seeks to compare broadcasters' speech rate and the number of pauses in 40 news bulletins from the BBC (United Kingdom), Radio France (France), RAI (Italy), and RNE (Spain). Most authors addressing the medium of radio recommend a speech rate of between 160 and 180 words per minute (wpm). If this rate is considered, only one radio station, $B B C$, would be within the suitable limits. Instead, higher speeds and fewer pauses have been identified in the RAI and RNE bulletins. The second part of this study attempts to analyze whether perception in the news can be affected by different speech rates. The findings indicate that the extent to which the individuals surveyed experience subjective assessment varies according to the speech rate.
\end{abstract}

Keywords: radio; speech rate; pauses; bulletins; news; perception.

\section{Introduction}

Radio expresses words through voices: voices dissolve into the air because radio language is evanescent. Sounds primarily exist in time. Whatever information the listener has missed or could not understand cannot be re-read. As Laurence Gilliam of BBC Radio states, radio is the art of communicating meaning at first hearing. Even though new technologies, and most notably the Internet, make it possible to re-play a radio program, few people actually take the trouble to listen again to an extract when they have experienced problems of comprehension. If it is necessary for the listener to understand the message upon the first hearing, whereby the voice is the principal element, it is possible to conclude that appropriate use of voice and style is essential in the radio communication process: "the style of newsreading is as important as the stories you 
choose to run and how they are written" (Chantler and Stewart 2003: 83). In this respect, one of the most important elements that determines the use of voice on the radio and the listener's understanding is speech rate, especially in news programs, based only on data. Speech rate, determined by speed and pauses, is an important factor in establishing clarity and intelligibility. If it is too fast, that is, with many words per minute and few pauses, the listener cannot understand the message upon the first hearing, particularly because a fast speech rate affects the speaker's diction. "En ce sens, parle vite est une des causes de la diction déficiente" (Bellenger 2001: 62), ${ }^{1}$ or as Karpf (2006: 43) points out, "You might think that, because speaking quickly makes speech less clear, it also makes it less persuasive." Thus, appropriate speech rate is an essential condition in radio news, more than in an entertainment program, because the listener receives a great deal of data in a short time. However, the important aspect to consider is that fast speech rate prevents the listener from assimilating the information because radio operates entirely without images and on the basis of a short-term memory having limited capacity, a difficulty which does not occur when words are accompanied by images (Campanella and Belin 2007; McGurk and MacDonald 1976). Therefore, the listeners need time to retain the information in their memory and with radio they have neither a visual support nor a second opportunity. If many words are pronounced without pauses over a short space of time, comprehension becomes difficult (Berlyne 1960).

On the other hand, in theory at least, slow speech rate should make it easier to assimilate the information in a certain message since it allows the listener more time to process the message. A slow speech rate with pauses allows a listener extra time for the processing of speech (Murphey et al. 2003). But if the speech rate is too slow, the listener's attention may be adversely decreased. Consequently, appropriate use of this element depends on the audience paying attention to the message. "In broadcasting, control of rate is important and reveals your sense of involvement and interest in a story" (Utterback 2000: 141). A presentation that unfolds at a slow pace can lead to it being difficult to maintain attention effectively (Berlyne 1960) and can result in weakened comprehension (Mastropieri et al. 1999), because an increase in the flow of information can raise attention and learning (LaBarbera and MacLachlan 1979).

\section{Speech rate in radio bulletins}

The speed of the actual articulation movements producing speech sounds occupies a very small range of variation between 4.4 to 5.9 syllables per second (Goldman-Eisler 1961: 171). Deese (1984) reports a normal speaking rate for conversational speech to be between 5 and 6 syllables per second. Differ- 
ences in speech rates between 4.4 and 5.9 syllables per second would be quite noticeable and should not be considered "a small range of variation" at all (Goldman-Eisler 1961: 171). This means that the average speech rate of adults in English is between 150 and 190 words per minute (Tauroza and Allison 1990), although in conversation this figure may rise considerably, reaching 200 wpm (Walker 2010; Laver 1994). Speech rate is a factor which depends partially on the language employed. A recent study reported different rates for different languages (Pellegrino et al. 2011): Japanese proved to be the language with the fastest rate with an average of 7.84 syllables per second, followed by Spanish (7.82), French (7.18), Italian (6.99), English (6.19), and German (5.97). These figures, however, refer to spontaneous speech. When, on the other hand, the speech is structured for use in public, as is the case in radio news broadcasts, the rate should be slightly slower, firstly because of the high data density, and secondly because the absence of images hinders the coding of information. For this reason, most authors addressing the medium of radio recommend a speech rate of between 160 and $180 \mathrm{wpm}$. According to Hills (1987), the most recommended pace is $160 \mathrm{wpm}$; McLeish (2005 [1978]: 71) sets the rate at between 160 and $180 \mathrm{wpm}$; Utterback (2000: 141) determines that a rate of between 145 and $180 \mathrm{wpm}$ is best: "Normally we read out loud at between 145 to $180 \mathrm{wpm}$. The most comfortable speed would be around 150 to 175 wpm." Boyd (2003) establishes the rate at between 140 and 220 wpm, although he considers $180 \mathrm{wpm}$ to be the most natural and pleasing rate.

Chantler and Stewart (2003: 87) share the same opinion: "the usual speed for reading on radio is three words a second." Kendall (2009), for radio interviews, reported a media speech rate of 5.34 syllables per second. In any event, Lawton (1930: 270) concluded that, "the only blanket rule which can justifiably be made with regard to the rate of delivery in radio speaking is that a speaker should talk as briskly as is consistent with good enunciation and proper employment of the Variety Stimuli." These data are supported by the work of Tauroza and Allison (1990) who analyzed the delivery rate of news items on BBC Radio and recorded a speed of between 150 and $170 \mathrm{wpm}$, moderately fast between 170 and $190 \mathrm{wpm}$, moderately slow between 130 and $150 \mathrm{wpm}$, faster than normal in $190 \mathrm{wpm}$, and on the contrary slower than normal in 130 wpm. These figures, in turn, reinforce the findings of Pimsleur et al. (1977) who recorded a delivery rate of between 160 and $190 \mathrm{wpm}$ for English and French radio news broadcasters, moderately fast between 190 and $220 \mathrm{wpm}$ and moderately slow between 130 and $160 \mathrm{wpm}$. In Spanish radio news, some research has shown that a high speech rate, of around $200 \mathrm{wpm}$, is used on all national radio stations (Rodero 2007).

Indeed, the data from various studies point to the existence of a determined prosodic style in radio news reading, certain of whose characteristics are shared by broadcasters from different countries (Nihalani and Lin 1998; 
Strangert 1991). One such pattern is precisely the tendency to read excessively fast and with a regular reading rate (Rodero 2007; Misono et al. 1991). This is a general trend among broadcasters because, as Utterback (2000: 140) states, "Rate is an area that many broadcasters find confusing. They feel they deliver their copy too slowly and when they speed up they get too fast." Although it is true that delivery speed depends on the language employed and, therefore, this must be taken into account, there is also a set of practices which comprise a particular news-reading style.

In any case, with regard to comprehension, it is very important to monitor this factor because many authors have found that recall and recognition of the news can be affected by speech rate (Meyerson 1974; Goldhaber 1974; Murphey et al. 2003). "Each aspect of fluency has a clear connection to text comprehension" (Hudson et al. 2005: 703). Lawton (1930) conducted research on students listening to radio speakers. In 34 cases, the speaker was deemed to have delivered the message too swiftly to be understood, while in 12 cases the delivery was too slow and, as a result, lacked interest. Rates of speech considered too slow were 120,124 , and $128 \mathrm{wpm} ; 135$ to $140 \mathrm{wpm}$ was considered a good average delivery. Borden (1927) came to the conclusion that a good average rate was $165 \mathrm{wpm}$, and that the delivery should contain certain marked variations in rate, while Lumley (1933) determined that the average syllable rate was in the region of 240 per minute and the word rate was 160 per minute. Similarly, in a test comparing four different rates, Nelson (1948) established that the most satisfactory rate was $175 \mathrm{wpm}$.

Along with speech, another aspect to consider is the number of pauses. Most studies have looked into the pattern of pauses and vocalizations as time unfolds in verbal situations, but they have concentrated on spontaneous speech (Dechert 1980; Goldman-Eisler 1968) and not on oral reading situations as the radio.

First, there are two types of pauses: filled and unfilled. The filled pauses are defined as a sound produced during speech that represents a silent period filled by a vocalization (umm, ehh). The unfilled pauses can be defined as the acoustical correlate for the perception of a silent portion in the speech signal, produced in conjunction, or not, with an inspiration, swallowing, any laryngophonatory reflex, or a silent expiration (Arim et al. 2003). Although in radio there are two types of pause, the first type is more suitable to structured texts, and so also to radio news presentations (Campione and Véronis 2005; Misono et al. 1991), whereas the second type is more characteristic of spontaneous speech.

Pauses have an important communicative function in radio because presenters chunk their discourse, using grammatical pauses which enable the listener to follow the syntactical organization and, thus, understand the information. In this sense, some authors indicate that the unfolding over time of patterns of vocalizations and pauses is distributed according to the syntax and 
semantic organization of the text (Goldman-Eisler 1968; Grosjean and Collins 1979; Lieberman 1969; Breznitz 1989). These pauses, which occur at grammatical junctions in the discourse, also allow the presenter to breathe. Cecot (2001) denominates as segmentation pauses those whose main function is the syntactical organization of the discourse and which are also exploited by the presenter to catch breath. Yet there is another type of pause which aids in reinforcing listener comprehension by distinguishing relevant and new data in the information (Hargrove and McGarr 1994). Since this type of pause helps the listener to differentiate between new and important data from among less relevant or secondary data, they have the effect of reinforcing comprehension (Giannelli 1992). Indeed, studies by O'Connell and Kowal (1972) have shown that pauses are vital in emphasizing the key elements of a message. Likewise, "pauses may serve not only to make time available for speaker's cognitive processes, but also to assist the listener in his task of understanding the speaker" (Butterworth 1980: 157). Rhetorical pause is the name given to those pauses which distribute information in the discourse according to communicative relevance and which, therefore, the speaker uses strategically (Cecot 2001). In addition to this function, such pauses can provide the listener with extra time to assimilate the information and allow for what is known as sub-vocal rehearsal, a brief review of the information received (Baddeley 1990). This gives the listener more time to cognitively process the information and so bring more resources to bear on the perceptual processing of the stimulus (Tanaka et al. 2011). For these reasons, when the task involves understanding a complex discourse such as a data-dense radio news item, it would be recommendable to employ not only segmentation pauses but to combine these with rhetorical pauses.

The studies of Arim et al. (2003) for spontaneous speech (radio interviews) concluded that the pause length averaged was $416 \mathrm{~ms}$, or $300 \mathrm{~ms}$ in Kendall's studies (2009). The shortest pause measured was $120 \mathrm{~ms}$ with a ratio of eight words per pause. Having analyzed a large corpus of read and spontaneous speech in five languages - English, German, French, Italian, and SpanishCampione and Véronis (2002) described a trimodal distribution of pauses, categorizing them as brief (200 ms), medium (200-1000 ms), and long (1000 $\mathrm{ms}$ ), the latter occurring only in spontaneous speech. Nevertheless, some research into the radio concludes that there are few examples of pauses and any such pauses have short durations. Grosjean and Collins (1979) have established the mean duration of the acoustic pause length in professional reading in $271 \mathrm{~ms}$ and the ratio of word per perceived pause in 12.2 words/pause. This is because when fluent readers deliver at a faster pace, they produce shorter and fewer pauses, at relatively long intervals (Misono et al. 1991; Grosjean and Collins 1979). "Professional announcing has few and short pauses with a multi-functional usage combining pausing with breathing, swallowing, etc. 
Pauses appear mainly at sentence boundaries where the theme of the message changes" (Gustafson-Capková and Megyesi 2001). Although the exact number and duration of these pauses may be conditioned by the language employed in each case, the authors describe an overall tendency toward a specific newsreading style. These authors consider that the reason for the short duration in the pauses in radio reading may be due to the fact that broadcasters are under time pressure. So these pauses are not strategically rhetorical silences but only segmentation pauses. "Silent pauses are rare and when they occur they are in connection to sentence boundaries and theme continuation" (GustafsonCapková and Megyesi 2001). In reality, broadcasters do not usually use pauses in a strategic communicative way with rhetorical function; rather, they use them to segment the news and, in particular, for physiological reasons. In this latter case, pauses are usually vocal noises: mainly inhalations. In GustafsonCapková and Megyesi's study (2001), 70\% of the pauses were with breathing or swallowing usually at the beginning of the sentence.

In sum, given the significance of speech rate in presenting news on the radio, conducting a study into this element of prosody seems entirely appropriate. Accordingly, this research is designed with a twofold aim. Firstly, it seeks to determine and compare broadcasters' rate of speech and the number of pauses made in 40 news bulletins from the BBC (United Kingdom), Radio France, RAI (Italy), and RNE (Spain). The objective is to assess whether speech rate and pauses unfold at suitable levels. Secondly, the aim is to analyze whether subjective assessment in the news can be affected by different speech rates.

\section{Method}

\subsection{First study}

This research attempts to quantify the speech rate and pauses in ten news bulletins from the BBC (United Kingdom), Radio France (RF), RAI (Italy), and RNE (Spain) to characterize this aspect in four countries representing western European broadcast news. The corpus for this research is formed by forty radio news bulletins. These bulletins have been selected as a unit of analysis because these news programs are brief, they summarize all the events "and bring the audience up-to-date with the latest events, and this means that analysis pieces or longer interviews are generally not included" (Fleming 2002: 104).

Radio stations only broadcasting news programs have been chosen (without having a music format). Therefore, the bulletins of the BBC World Service, Radio France International, RAI International, and RNE World Service have been analyzed. Given that they address an international audience which may not have a fluent command of the broadcasting language, the world service 
news programs have been considered because they are expected to be clearer than local news programs.

The corpus was obtained by recording the radio stations in six weeks during May and June 2010. Ten bulletins were collected from each radio station. Each bulletin lasts between 10 and 15 minutes. Overall, all of the portions of each news presenter's delivery have been selected. This means that, through soundediting software, we only extracted the parts in which the news presenter intervenes and omitted the contributions from other broadcasters or external sources. In a news bulletin, it is the news presenters who set the pace as they act as the medium for conveying the information. Moreover, all the presenters selected were male broadcasters so as to avoid introducing another variable to the study as would be the type of voice. Since less deep sounds are more intelligible, typically the case with a woman's voice, some bulletins might seem more comprehensible than others. As a result, only male broadcasters were employed to avoid this variable occurring. Future studies will analyze bulletins presented by female voices. Lastly, speech rate, pauses, and their relationship have been analyzed in fifty sound files (between 30 seconds and 1 minute) for each radio station. This variation in the duration depends on the contents of the news item. Within a particular news bulletin, not all the items are of the same length. For the selection in this study we used the Adobe Audition professional editing software.

Speech rate was obtained by counting the words per minute (wpm)/total time, as Franke (1939) and Darley (1940) recommended. The main reason for using this calculation is that the majority of authors who have studied the radio medium use this form of measurement, which is also of common use within the radio industry. By adopting this method, therefore, later comparisons would be easier to carry out. However, in order to facilitate the comparison with other investigations, this study also includes the measure syllables per second $(\sigma / s)$ in accordance with a number of other authors that use this unit (Kendall 2009; Salmons et al. 2008; and often Miller et al. 1984). In calculating the words the clitics were included in all the languages. To carry out the measurement, we first transcribed all the recordings and then counted the words pronounced for each time unit (a measurement given by the sound-editing software).

Pauses were counted using two parameters: pauses per minute and the length of each pause. Lastly, the frequency of the pauses compared to the number of words was analyzed. The Praat voice-analysis software was used to obtain measurements (Boersman and Weenink 2010). These complex and unfilled pauses are the only ones occurring in this study, as on radio (Campione and Véronis 2005). Since it was a discourse written to be read, there were logically no filled pauses. Taking into account that Grosjean and Collins's study (1979) points out that radio professionals make short pauses, and with a view to comparing our results with the said study, we decided that only pauses longer than 
100 ms would be included. These short pauses, as Campione and Véronis (2005) have proved, are never demarcative and they often are of a respiratory nature.

\subsection{Second study}

Having established the general parameters for the pace of the bulletins broadcast by these four networks, the aim of the second part of this study is to ascertain whether the speech rate can affect the subjective assessment of the news.

A random sample of 60 Erasmus students (English, French, Italian, and Spanish; 40 female and 20 male) was selected for this goal. The overall sample was divided again at random into four groups of 15 people (one group per language), keeping the gender balance.

- The bulletin of the BBC was listened to by the English group (15 students).

- The bulletin of the Radio France was listened to by the French group (15 students).

- The bulletin of the RAI was listened to by the Italian group (15 students).

- The bulletin of the RNE was listened to by the Spanish group (15 students).

Each group listened to each of the four bulletins in similar and optimal conditions with regard to sound. As an initial instruction they were merely informed that they were going to listen to a radio bulletin regarding which they would subsequently be required to answer some questions.

Once the bulletins had been listened to, the questionnaire was handed out in order to assess the perception of the news. The aim of the questionnaire was to examine the extent to which the subjects in the sample were able by themselves to perceive the speech rate of the bulletin. The questionnaire included two questions on subjective assessment where the sample was asked to evaluate the speaker's speech rate. To do so, a five-point scale was established where respondents had to state whether they considered it was very fast (5), fast (4), normal (3), slow (2), or very slow (1). Then, the second question was intended to ascertain whether this speech rate of delivery was conducive to understanding the news, again where a five-point scale was provided to show whether understanding was considered to be very difficult (5), difficult (4), normal (3), easy (2), or very easy (1). In both cases, five degrees of options were employed, given the difficulty of establishing a greater number of possible choices of delivery speed and the risk of excessively complicating the levels of understanding. Excessive segmentation in establishing the levels of difficulty would oblige the experimental subjects to use too fine an approach in forming their answers, leading them to add nuances that were irrelevant to the aim of the study. 
Table 1. Means and standard deviations of radio stations

\begin{tabular}{|c|c|c|c|c|c|c|c|c|}
\hline \multirow[t]{2}{*}{ Station } & \multicolumn{2}{|c|}{ Speed rate (wpm) } & \multicolumn{2}{|c|}{$\begin{array}{l}\text { Pauses per } \\
\text { minute }\end{array}$} & \multicolumn{2}{|c|}{$\begin{array}{l}\text { Length of } \\
\text { pauses } \\
\text { (milliseconds) }\end{array}$} & \multicolumn{2}{|c|}{$\begin{array}{l}\text { Words per } \\
\text { pause }\end{array}$} \\
\hline & Mean & $\mathrm{SD}$ & Mean & $\mathrm{SD}$ & Mean & $\mathrm{SD}$ & Mean & $\mathrm{SD}$ \\
\hline RNE & 209.96 & 22.17 & 8.60 & 3.08 & 110 & 0.05 & 23.92 & 7.36 \\
\hline RAI & 192.46 & 15.39 & 9.87 & 2.55 & 146 & 0.06 & 20.08 & 4.62 \\
\hline $\mathrm{RF}$ & 188.93 & 12.90 & 10.47 & 2.47 & 153 & 0.05 & 18.63 & 3.58 \\
\hline $\mathrm{BBC}$ & 167.54 & 11.67 & 11.47 & 2.69 & 169 & 0.07 & 18.61 & 3.58 \\
\hline
\end{tabular}

\section{Results}

\subsection{First study}

The overall results for the four radio stations were analyzed, followed by the data for each item: speech rate, pauses, and the relationship between them. General data corresponding to RNE, RF, RAI, and BBC are set out in Table 1.

An analysis of variation by means of an ANOVA shows that significant differences are evident for all the variables analyzed: speech rate $(F(3,49)=33.86$, $P<0.000)$, pauses $(F(3,49)=9.78, P<0.000)$, length of pauses $(F(3,49)=$ $6.13, P<0.001)$, and words per pause $(F(3,49)=12.39, P<0.000)$. The data clearly show that Mediterranean radio stations - RNE and RAI - have the highest speech rate, $\mathrm{RF}$ is in the middle, and the $\mathrm{BBC}$ in particular has a lower speech rate. The only radio station with a speech rate of more than $200 \mathrm{wpm}$ is the Spanish radio station RNE $(6.99 \mathrm{\sigma} / \mathrm{s})$, although the Italian radio station, RAI, is close to this limit $(6.41 \sigma / \mathrm{s})$. Slower speech rates are manifested by the French radio station $\mathrm{RF}(6.29 \sigma / \mathrm{s})$ and the English radio station from the BBC $(5.93 \sigma / s)$.

As can be seen, the values are similar to those obtained by Pellegrino and his colleagues (2011) — Spanish (7.82), French (7.18), Italian (6.99), and English (6.19) - though in all cases they are slightly lower, as is to be expected from a discourse in a medium such as radio. The smallest differences occur between spontaneous speech and radio news in English, followed by Italian.

Post-hoc tests for these means, specifically Tukey's test, show that the differences are substantial in all areas where RNE has been compared to the other broadcasters $(P<0.000)$ and for all areas where the BBC is compared to the other broadcasters: RNE and RAI $(P<0.000)$ and indeed RF $(P<0.005)$. The data are shown in Figure 1.

If the pauses are closely examined, the results can be presented in the following order of radio stations. Firstly, RNE broadcasters produce the least 


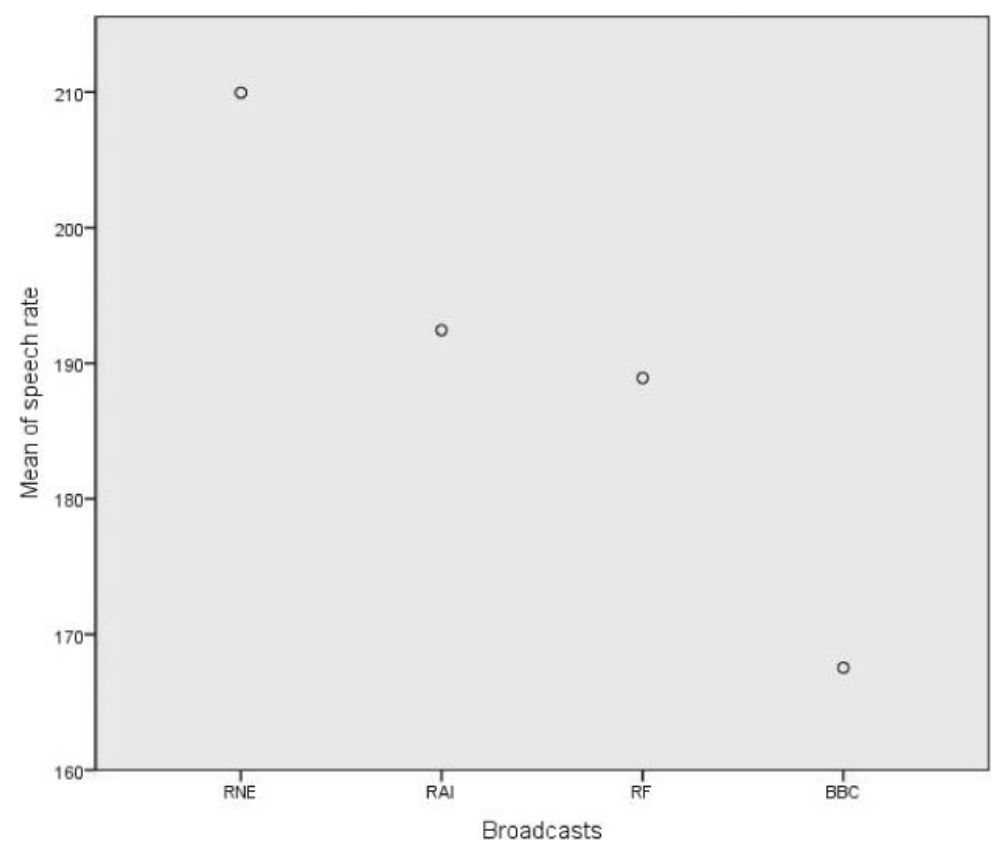

Figure 1. Broadcast speech rate

number of pauses: $8.6 \mathrm{ppm}$ (pauses per minute). Not far away are the RAI broadcasters who have a mean of $9.87 \mathrm{ppm}$. The French broadcasters have a mean of $10.47 \mathrm{ppm}$. And, finally, the broadcasters with the greatest number of pauses are from the BBC with 11.47 ppm, as is shown in Figure 2.

Post-hoc tests on these measures, in particular Tukey's test, show that the differences are significant if we compare RNE on the one hand with RF $(P<0.004)$ and the BBC $(P<0.000)$ on the other.

Owing to physiological reasons, all the pauses analyzed in this research have involved breathing. These were pauses produced between phonic groups whose functions are to organize discourse syntactically and to allow the presenter to breathe. In the corpus, according to some studies addressing the issue of pauses on the radio, no strategic rhetorical pauses were made. In all instances examined, pauses were vocal noises, primarily inhalations, which were clearly perceived by the listener. In fact, the presenters' intakes of air are perfectly audible and visible in the sound editor, as can be seen in the following example of a complete phrase from a BBC news item (Figure 3).

The radio stations maintain the same order when it comes to the number of pauses and speech rate. This means that speech rate is related to pauses. When a broadcaster maintains a high speech rate, he also makes fewer pauses 


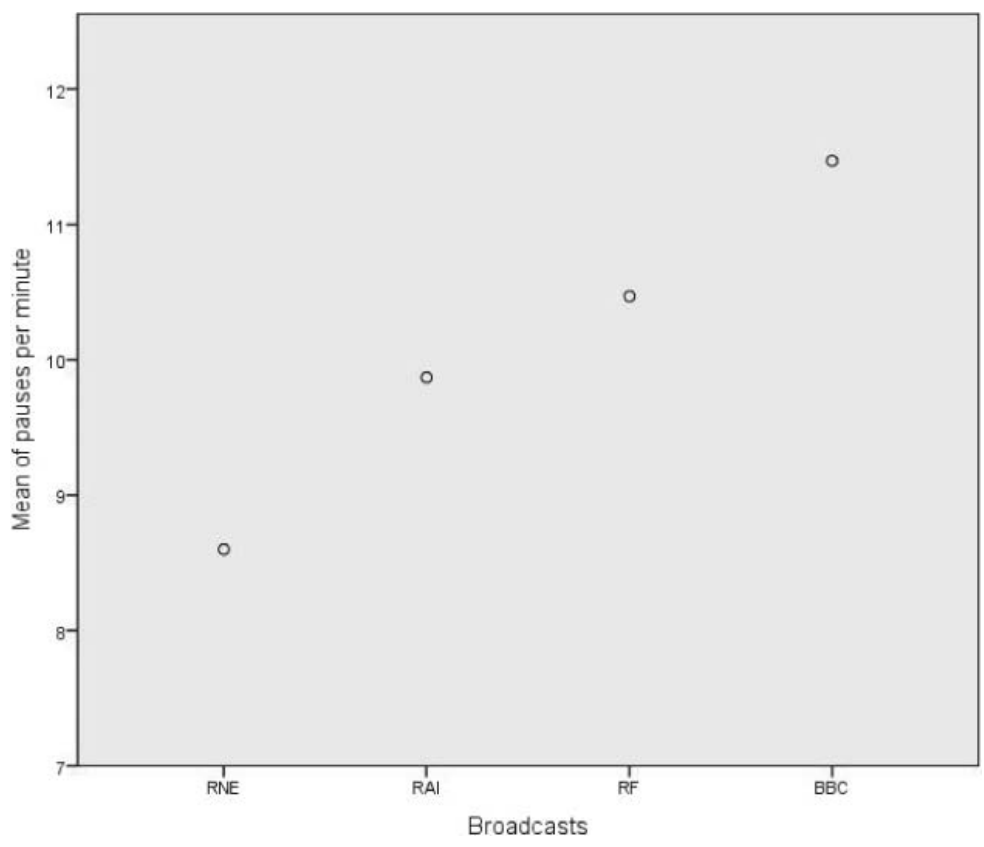

Figure 2. Broadcast pauses

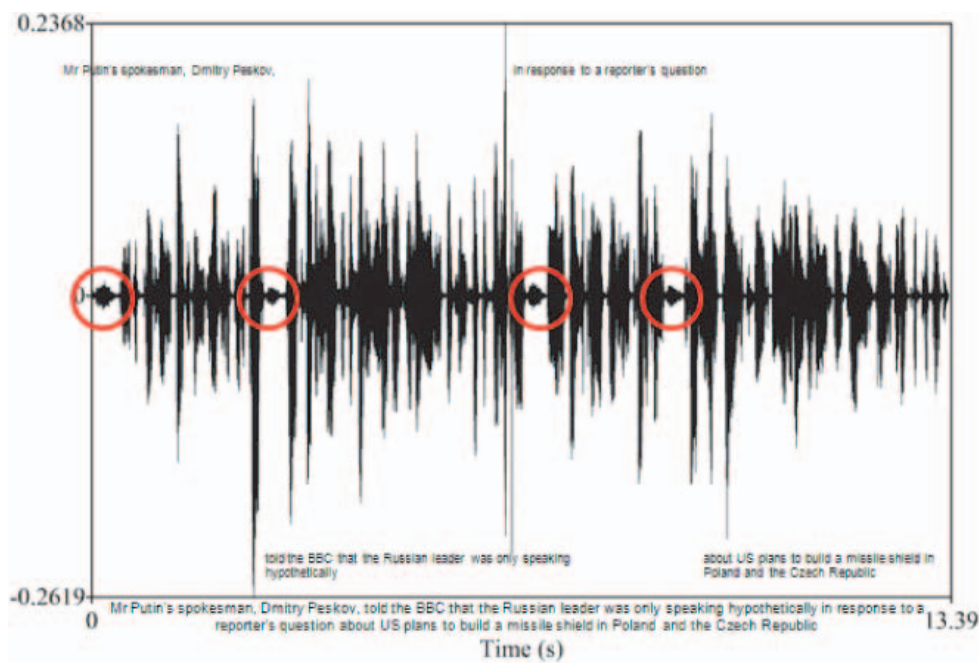

Figure 3. Distribution of pauses in a BBC sentence 
per minute, as suggested by Grosjean and Collins (1979). Indeed, there is a significant inverse correlation between these variables when measured using Pearson's correlation ( $r=-0.207$, Sig. (2-tailed): 0.003). Accordingly, broadcasters on the BBC have more pauses in their speeches while Spanish broadcasters use fewer pauses. At the same time, this means that speech that is delivered at a faster rate, on RNE for instance, is still perceived at a swifter rate by the listeners because of the lower number of pauses used (Misono et al. 1991); whereas the general pace is considered slower, with the $\mathrm{BBC}$ for example, when a slower speech rate is combined with more pauses. Because the pauses in this study are segmentation pauses and not rhetorical pauses, these differences can be explained by the paragraph structure of each language. Shorter paragraphs mean more paragraphs which mean more frequent pauses and longer pauses.

In fact, data on the length of pauses bear the same order with regard to the radio stations. Pauses with a shorter duration are made on RNE (110 ms) and on RAI (140 ms). By contrast, longer pauses are on RF $(150 \mathrm{~ms})$ and the BBC $(160 \mathrm{~ms})$. Nevertheless, these pauses are very brief in accordance with the findings described in Campione and Véronis (2002), in Grosjean and Collins (1979), and in Kendall (2009). The differences between the stations are minimal except when the two stations at either end of the spectrum are contrasted. Tukey's test reveals that the differences are significant when RNE is compared to the BBC $(P<0.001)$. Figure 4 shows these data.

Lastly, the relationship between words and pauses is also analyzed. This determines the number of words pronounced before each pause. These data demonstrate that broadcasters on the $\mathrm{BBC}$ pronounce an average number of 18 words for each pause (that is, that they pause after every 18 words); broadcasters on RF utter a mean of 18 words per pause; those on RAI utter a mean of 20 words per pause; and, lastly, broadcasters on RNE pronounce 24 words per pause. Post-hoc tests for these mean values show that the differences are significant for all cases when RNE is compared to RF, the BBC $(P<0.000)$ and RAI $(P<0.001)$. Figure 5 shows these data.

In conclusion, the pauses analyzed in this study were few in number, less than the 12.2 words/pause established by Grosjean and Collins (1979) in professional speech, and briefer in terms of length than $270 \mathrm{~ms}$. RAI and RNE obtained the worst results, according to the studies conducted by Grosjean and Collins (1979) and Gustafson-Capková and Megyesi (2001). Therefore, the best structured message (with slower speech rate and more pauses) is that produced by the BBC and RF. For every 18 words there is one pause, which allows the listener to assimilate the information better. That is because slow speech rate with pauses allows a listener extra time for the processing of speech, thus one would expect to have higher intelligibility scores. However, on RAI ( 20 words per pause) and RNE ( 24 words per pause) the messages have 


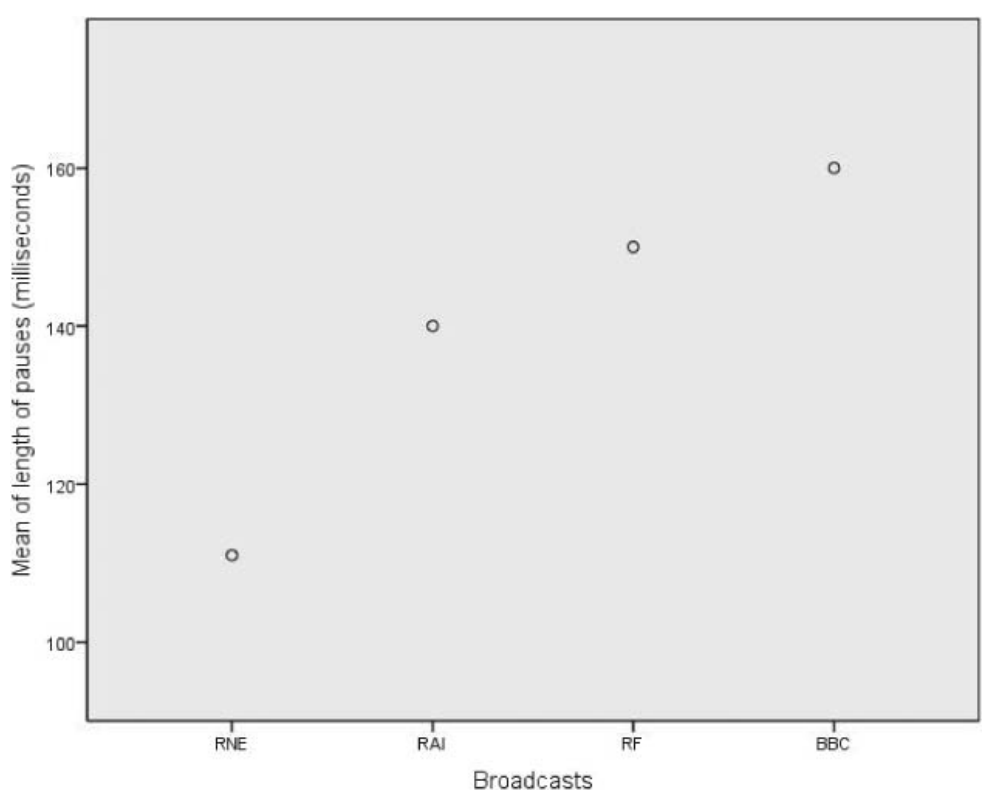

Figure 4. Broadcast length of pauses

a greater number of words for each pause. This produces a highly incessant speech with no differences between the significant and the supplementary parts, preventing the listener from being allowed time in order to retain the information in their memory. The explanation is straightforward. As is shown in Figure 6, the BBC presenters distribute the information periods with more pauses, which make the text more segmented. This means that the phonic groups are more clearly differentiated and these, as oral units of meaning, enable the listener to better distinguish the data and assimilate them more efficiently. In contrast, the discourse of the RNE presenters, with hardly any pauses, produces an undifferentiated, continuous expression with scarce distribution of the information among the phonic groups, which hinders listener comprehension.

\subsection{Second study}

Having carried out this prior analysis, the second part of this study sought to verify whether the level of perception of a radio bulletin could be affected by the pace of delivery. In order to assess this objective in the four bulletins (BBC, RF, RAI, and RNE), an ANOVA was performed on the two dependent 


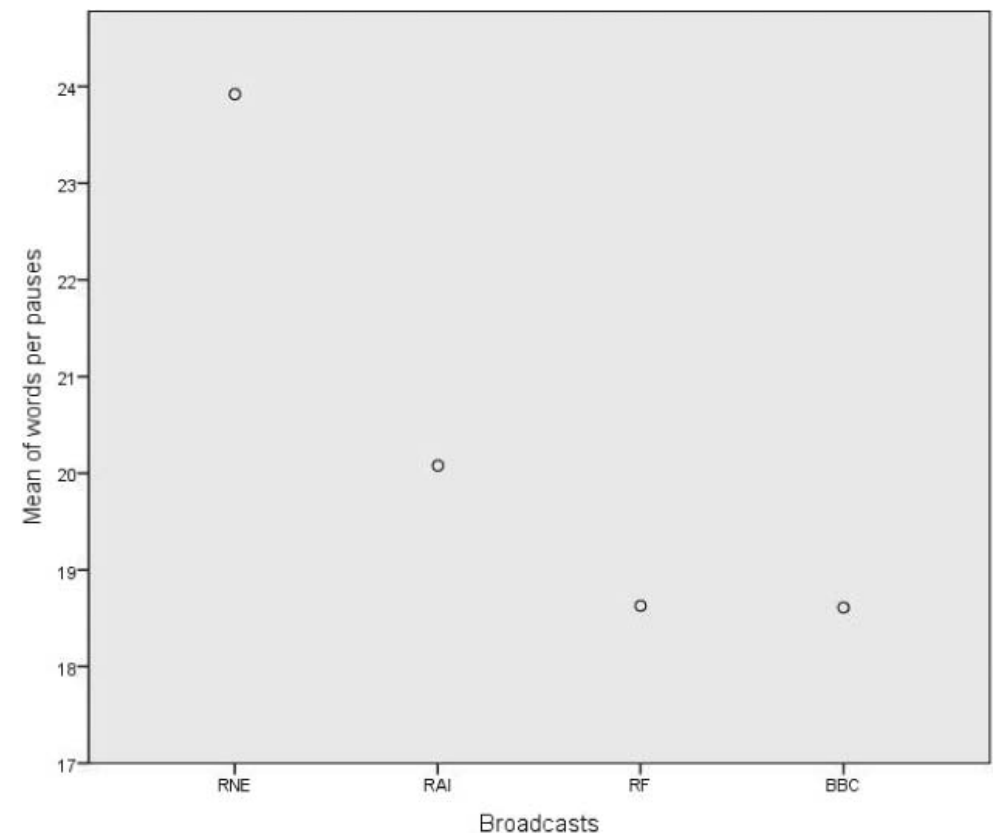

Figure 5. Broadcast words per pause

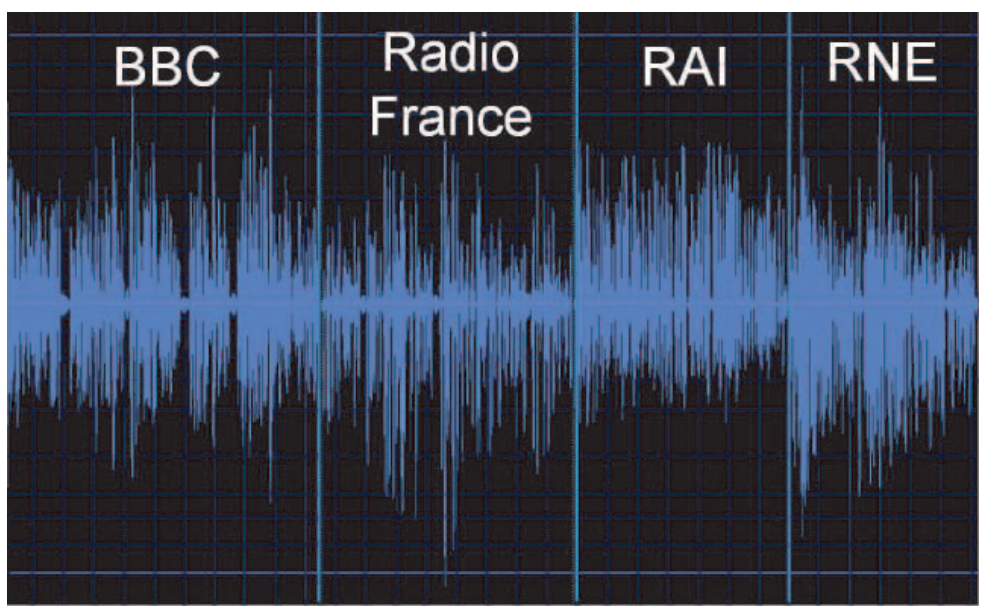

Figure 6. Broadcast distribution of speed and pauses 
Table 2. Means and standard deviations of perception

\begin{tabular}{llllll}
\hline Bulletin & \multicolumn{2}{c}{ Speech rate } & & \multicolumn{2}{c}{ Difficulty } \\
\cline { 2 - 3 } & Mean & SD & & Mean & SD \\
\hline BBC & 2.75 & 0.63 & 1.85 & 0.98 \\
RF & 3 & 0.72 & 2.45 & 0.99 \\
RAI & 3.45 & 0.68 & 3.60 & 0.82 \\
RNE & 4.15 & 0.93 & 4.10 & 0.64 \\
\hline
\end{tabular}

Speech rate: very fast (5), fast (4), normal (3), slow (2), or very slow (1)

Difficulty: very difficult (5), difficult (4), normal (3), easy (2), or very easy (1)

variables: subjective assessment of speech rate and subjective assessment of difficulty. Using an alpha level of 0.001 to evaluate homogeneity assumptions, Box's $M$ test on homogeneity of covariance was not significant $(P=0.456)$. Levene's homogeneity of variance test was statistically insignificant (subjective assessment of speech rate: $P=0.121$; subjective assessment of difficulty: $P=0.218$ ) making it possible to assume that the variations were the same and fell in line with the principle of homoscedasticity. General data corresponding to RNE, RF, RAI, and BBC are set out in Table 2.

The aim of the first part of the questionnaire was to examine the extent to which the subjects in the sample were able by themselves to perceive the speech rate of the bulletin. For the four cases, the differences were statistically significant $(F(3,56)=13.26, P=0.000)$. The pairwise comparisons show that the differences are only significant for BBC and RNE $(P=0.000)$. The results in response to this question show that both $\mathrm{BBC}$ and RF bulletins were considered to be normal; the RAI bulletin was considered between normal and fast; and, lastly, RNE was closest to receiving the fastest rating.

In relation to the second question where the individuals had to classify how easy the bulletin was to understand, the differences were statistically significant $(F(3,56)=26.92, P=0.000)$. The pairwise comparisons show that the differences are significant for all comparisons made, except when comparing $\mathrm{BBC}$ and RF bulletins, which show similar values. Therefore, the BBC bulletin was easy to understand, the RF bulletin was considered as normal, the RAI bulletin was between normal and difficult to understand, and, lastly, the RNE bulletin was considered the most difficult to understand.

These data lead us to conclude that the extent to which the individuals surveyed experience subjective assessment varies according to the speech rate. Bulletins having a pace considered to be normal (BBC and RF) were considered the easiest to understand. On the other hand, those that were faster (RAI and RNE) were the most difficult to understand, especially RNE, as shown in Figure 7. 


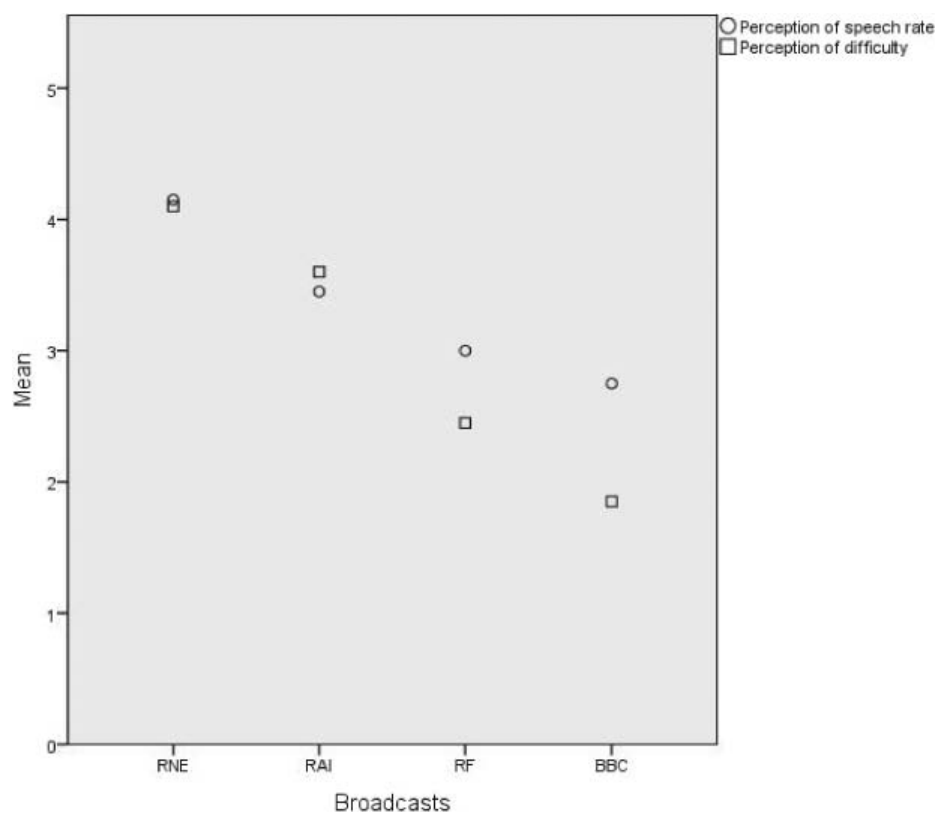

Figure 7. Perception of speech rate and difficulty

\section{Discussion and conclusion}

Speech rate is one of the most important elements of voice on the radio. A listener's understanding depends to a large extent on the speed and pauses that broadcasters make in their message. A high speech rate with few pauses can affect the listener's potential to understand the information. This is sufficient cause in order to give serious consideration to speech rate and to the reading speed and pauses adopted.

Following analysis of these elements, only one radio station, BBC, would be within the suitable limits, with $167 \mathrm{wpm}$, although RF, with $189 \mathrm{wpm}$, would be close to the recommendable rate, as per the findings of previous studies. Considering the results of Pellegrino's study (Pellegrino et al. 2011), in which French is the second fastest language of those analyzed in this study, it may be concluded that it is not an excessive speed. In fact, the results of the present study of perception show that natives of these two languages did not experience comprehension difficulties when they listened to them. Nevertheless, RAI and RNE have very high speed rates, with 192 and $210 \mathrm{wpm}$, respectively, especially RNE, surpassing $200 \mathrm{wpm}$. Some Spanish investigations confirm these values and they locate the RNE speed rate between 210 and $230 \mathrm{wpm}$, in 
accordance with the findings of Rodero (2007). These findings can be explained because in Mediterranean countries, especially in Spain, speech is faster than in other countries, as Pellegrino et al. (2011) have shown. But, in any case, this speed rate is considered as excessive for a media like radio, although Spanish and Italian listeners are accustomed to a faster speech. In fact, the results of this study of perception clearly show that there is an imbalance between the expected speech rate in radio news with regard to the speed of the spoken language and the speed which actually occurs in everyday practice. This poses a very real problem which the radio industry would do well to address. This cannot be ignored: if the broadcaster wants to be truly effective in communicating news on radio, he has to use a slower speed rate, similar to that on RF and especially that on BBC.

Along with speech, the other aspect considered in this study is the number of pauses. The pauses analyzed in this study were few in number, less than the 12.2 words/pause established by Grosjean and Collins (1979) in professional speech. Instead, the pauses were briefer in terms of length than $270 \mathrm{~ms}$, in accordance with the findings described by Campione and Véronis (2002) and Kendall (2009). RAI and RNE obtained the worst results, affirming the findings by Grosjean and Collins (1979) and Megyesi and Gustafson-Capková (2001). Also, when a broadcaster maintains a high speech rate, he also makes fewer pauses per minute.

Likewise, these pauses have been motivated by the need to breathe; therefore, the pauses have not been strategically determined silences or ones having a certain rhetorical function. The broadcasters analyzed have not used pauses in a strategic way, for example, to highlight a particular idea or piece of data and distinguish it from secondary information. The pauses in this study have merely been those necessary for syntactical segmentation and, in particular, for the presenter to catch breath. Thus, all pauses in this study have been vocal noises: mainly inhalations. It is advisable for broadcasters to breathe correctly in order to avoid these frustrating noises, especially when it comes to gasping. They are recommended to inhale in shallow draughts. On the basis of the findings of the present study, this recommendation can be applied to all radio stations.

Finally, the conclusion to be drawn from the first part of the study is that the $\mathrm{BBC}$ has the radio station with the most appropriate general speech rate, allowing for a better understanding on the part of listeners not only on account of the fact that the speech rate adopted is within recommended values, but also because the $\mathrm{BBC}$ had more, longer pauses per minute. As a result, information from $\mathrm{BBC}$ can be considered formally better structured, as it is shown in Figure 6. By combining deliberate pace with pauses, BBC provides access to fresh data in a better way, ensuring that the listener does not perceive problems of comprehension. After the $\mathrm{BBC}, \mathrm{RF}$ is the network that obtains the best results. 
The speech rate adopted is a little higher though it improves with the number of pauses and the length thereof. Accordingly, the message conveyed is not perceived as being too fast and the listener does not perceive difficulties of comprehension

Nevertheless, the other two radio stations - RAI and RNE - have higher values than their English and French counterparts, especially in the case of Spain. Firstly, RAI broadcasters pronounce 192 wpm with few and shorter pauses per minute, meaning that the message is perceived as more accelerated. Secondly, the values for RNE are also somewhat negative. The speech rate of this latter network is the highest found in this study: $210 \mathrm{wpm}$, a pace at which the listener claims to perceive difficulties of comprehension. Similarly, it is the radio station in which fewer pauses with a shorter length are produced per minute. It is important not to overlook the fact that, if the broadcaster wants to be truly effective in communicating news on radio, he must use a moderate speech rate, similar to that used on RF and especially that of the BBC. This is an aspect that should be reviewed, as indeed the data from the second part of this study confirm.

The results of the second part of this study back the findings of previous research in which a relationship has been established between the speech rate of delivery and the level of perception when applied to radio news, as is the case we are dealing with. As several authors have ascertained (Meyerson 1974; Goldhaber 1974; Hudson et al. 2005; Goldstein 1940; Lawton 1930; Borden 1927; Lumley 1933; LaBarbera and MacLachlan 1979; Nelson 1948), the data illustrate that subjective assessment on the part of the individuals surveyed matches the findings obtained when it comes to understanding. Bulletins perceived as having a normal pace (BBC and $\mathrm{RF}$ ) and considered as easier to understand were recalled to the greatest extent. However, those that were perceived as swifter (RAI and RNE) were assessed as difficult to understand.

In conclusion, if we consider that a key goal of radio communication is for the listener to understand the news, it is clear that the results of this study have significant repercussions in the field of teaching and research into media studies, and specifically, on radio communication.

\subsection{Limitations and future research}

Although the findings of this research provide significant results on the speech rate applied to radio news bulletins, the data must be interpreted within the limits of a laboratory-style study.

Firstly, the study is carried out using individuals and focusing on listening to a radio bulletin, though in practice this is not the usual, natural manner in which most listeners hear the radio. Radio often entails people doing other 
activities while listening; hence, the listener is rarely exclusively paying attention to the radio.

Secondly, the findings of this study can only be applied to the international service radio bulletins on the stations analyzed. This news service bears different characteristics to those usually found on local broadcasts because it is intended for an international audience. It is also important to draw attention to the fact that the character of delivery of each broadcaster varies as it is part of a particular style. As a consequence, a sample chosen from differing broadcasters may bring about variations in the findings for the variables examined.

\section{Note}

1. In this sense, speaking fast is one of the causes of the poor diction.

\section{References}

Arim, Eva, Francisco Costa \& Tiago Freitas. 2003. An empirical account of the relation between discourse structure and pauses in Portuguese. Paper at Prosodic Interfaces, Acoustique, Acquisition, Interpretation, Nantes. http://www.iltec.pt/pdf/wpapers/2003-prodip-disc_pauses.pdf.

Baddeley, Alan D. 1990. Human memory. Theory and practice. London: Lawrence Erlbaum.

Bellenger, Lionel. 2001. L'excelence à l'oral. Issy-les-Moulineaux: ESF.

Berlyne, Daniel E. 1960. Conflict, arousal and curiosity. New York: McGraw-Hill.

Boersman Paul \& David Weenink. 2010. Praat: Doing phonetics by computer. Version 5.3.04. http://www.fon.hum.uva.nl/praat.

Borden, R. C. 1927. The principles of effective radio speaking. Modern Eloquence XV. 9-17.

Boyd, Andrew. 2003. Broadcast journalism. Techniques of radio and television news. Oxford: Focal Press.

Breznitz, Zvia. 1989. Vocalization and pauses in fast-paced reading. Journal of General Psychology 117(2). 153-159.

Butterworth, Brian. 1980. Evidence from pauses in speech. In Brian Butterworth (ed.), Language production, vol. 1: Speech and talk, 155-176. San Diego: Academic Press.

Campanella, Salvatore \& Pascal Belin. 2007. Integrating face and voice in person perception. Trends in Cognitive Sciences 11(12). 535-543.

Campione, Estelle \& Jean Véronis. 2002. A large-scale multilingual study of silent pause duration. Paper at Speech Prosody 2002, ISCA, Aix-en-Provence, France, 11-13 April. http://citeseerx. ist.psu.edu/viewdoc/summary?doi=10.1.1.12.561.

Campione, Estelle \& Jean Véronis. 2005. Pauses and hesitations in French spontaneous speech. Proceedings of DiSS'05, Disfluency in Spontaneous Speech Workshop, 10-12 September 2005, 43-46. Aix-en-Provence, France: ISCA. http://www.isca-speech.org/archive_open/archive papers/diss_05/dis5_043.pdf.

Cecot, Michela. 2001. Pauses in simultaneous interpretation: A contrastive analysis of professional interpreters' performances. The Interpreters' Newsletter 11. 63-85.

Chantler, Paul \& Peter Stewart. 2003. Basic radio journalism. Oxford: Focal Press.

Darley, F. L. 1940. A normative study of oral reading rate. Ames, IA: State University of Iowa Master's thesis. 


\section{Emma Rodero}

Dechert, Hans. 1980. Temporal variables in speech. New York: Mouton.

Deese, James. 1984. Thought into speech: The psychology of a language. Englewood Cliffs, NJ: Prentice-Hall.

Fleming, Carole. 2002. The radio handbook. New York: Routledge.

Franke, Phyllis Elizabeth. 1939. A preliminary study validating the measurement of oral reading rate in words per minute. Ames, IA: State University of Iowa M.A. thesis.

Giannelli, Luciano. 1992. Sul valore comunicativo delle pause "vuote" nella narrazione e nel proverbio, nella prospettiva funzionale della frase. In E. Cresti, N. Maraschio \& L. Toschi (eds.), Storia e Teoria dell'Interpunzione, 311-354. Rome: Bulzoni Editore.

Goldhaber, Gerald M. 1974. Effects of speech compression training in comprehension of native speakers of English and Navajo. In Sam Duker (ed.), Time compressed speech: An anthology and bibliography, 730-735. Metuchen, NJ: The Scarecrow Press.

Goldman-Eisler, Frieda. 1961. The significance of changes in the rate of articulation. Language and Speech 4(4). 171-174.

Goldman-Eisler, Frieda. 1968. Psycholinguistics: Experiments in spontaneous speech. London: Academic Press.

Goldstein, Harry. 1940. Reading and listening comprehension at various controlled rates. (Contributions to Education 821). Columbia: Columbia University.

Grosjean, Francois \& Michael Collins. 1979. Breathing, pausing and reading. Phonetics 36. 99 113.

Gustafson-Capková, Sonia \& Beáta Megyesi. 2001. A comparative study of pauses in dialogues and read speech. Proceedings of Eurospeech 2001, vol. 2, 931-935. Aalborg, Denmark: ISCA. http://www.speech.kth.se/prod/publications/files/771.pdf.

Hargrove Patricia M. \& Nancy S. McGarr. 1994. Prosody management of communication disorders. San Diego, CA: Singular.

Hills, George. 1987. Los informativos en radiotelevisión (News on radio and television). Madrid: IORTV.

Hudson, Roxanne F., Holly B. Lane \& Paige C. Pullen. 2005. Reading fluency assessment and instruction: What, why, and how? The Reading Teacher 58(8). 702-714.

Karpf, Ann. 2006. The human voice. New York: Bloomsbury.

Kendall, Tyler S. 2009. Speech rate, pause, and linguistic variation: An examination through the sociolinguistic archive and analysis project. Durham, NC: Duke University Graduate School, Department of English dissertation.

LaBarbera, Priscilla \& James MacLachlan. 1979. Time-compressed speech in radio advertising. Journal of Marketing 43(1). 30-36.

Laver, J. 1994. Principles of phonetics. Cambridge: Cambridge University Press.

Lawton, Sherman Paxton. 1930. Principles of effective radio speaking. Quarterly Journal of Speech 16(3). 225-277.

Lieberman, Philip. 1969. Some effects of semantic and grammatical context on production and perception of speech. Language and Speech 6. 172-187.

Lumley, Frederick H. 1933. Rates of speech in radio speaking. Quarterly Journal of Speech 19(3). 393-403.

Mastropieri, M. A., A. Leinart \& T. E. Scruggs. 1999. Strategies to increase reading fluency. Intervention in School and Clinic 34. 278-283.

McGurk, Harry \& John MacDonald. 1976. Hearing lips and seeing voices. Nature 264. 746-748.

McLeish, Robert. 2005 [1978]. Radio production. Oxford: Focal Press.

Megyesi Beáta \& Sonia Gustafson-Capková. 2001. Pausing in dialogues and read speech in Swedish speakers: Production and listeners' interpretation. Paper at ITRW on prosody and speech recognition and understanding, Red Bank, NJ, 22-24 October. http://www.isca-speech.org/ archive_open/archive_papers/prosody_2001/prsr_020.pdf. 
Meyerson, Marion D. 1974. An exploration of comprehension differences in time-compressed Japanese, Chinese, Indi and English speech. In Sam Duker (ed.), Time compressed speech: An anthology and bibliography, 730-735. Metuchen, NJ: The Scarecrow Press.

Miller, Joanne L., Francois Grosjean \& Concetta Lomanto. 1984. Articulation rate and its variability in spontaneous speech: A reanalysis and some implications. Phonetica 41(4). 215-225.

Misono, Yasuko, Miyoko Sugito \& Shigeru Kiritani. 1991. Statistical characteristics of pauses in different speech styles. Annual Bulletin of the Research Institute of Logopedics 25. 75-80.

Murphey, Carol, Kathryn Dobie \& James Grant. 2003. Time versus pause manipulation in communications directed to the young adult population: Does it matter? Journal of Advertising Research 43. 281-292.

Nelson, Harold E. 1948. The effect of variation of rate on the recall by radio listeners of "straight" newscasts. Communication Monographs 15(2). 173-180.

Nihalani, Paroo \& Tai Poo Lin. 1998. Intonation patterns in news broadcasts. World Englishes 17(1). 15-29.

O'Connell, Daniel \& Sabine Kowal. 1972. Cross-linguistic pause and rate phenomena in adults and adolescents. Journal of Psycholinguistic Research 1(2). 155-164.

Pellegrino, François, Christophe Coupé \& Egidio Marsico. 2011. Across-language perspective on speech information rate. Language 87(3). 539-558.

Pimsleur, Paul, Charles Hancock \& Patricia Furey. 1977. Speech rate and listening comprehension. In M. Burt, H. Dulay \& M. Finocchiaro (eds.), Viewpoints on English as a second language, 27-34. New York: Regents.

Rodero, Emma. 2007. Characterization of a presentation on audiovisual media. Estudios del mensaje periodístico $13.523-542$.

Salmons, Joseph, Ewa Jacewicz \& Robert F. Allen. 2008. Fast talkers versus slow talkers: Speech rate across dialect, generation, and gender. Paper at American Dialect Society Annual Meeting, Chicago.

Strangert, Eva. 1991. Phonetic characteristics of professional news reading. Perilus 13. 39-43.

Tanaka, Akihiro, Shuichi Sakamoto \& Yòiti Suzuki. 2011. Effects of pause duration and speech rate on sentence intelligibility in younger and older adult listeners. Acoustic Society \& Technology 32(6). 264-267.

Tauroza, Steve \& Desmond Allison. 1990. Speech rates in British English. Applied Linguistics 11. 90-105.

Utterback, Ann S. 2000. Broadcast voice handbook. Chicago: Bonus Book.

Walker, Gareth. 2010. The phonetic constitution of a turn-holding practice: Rush-throughs in English talk-in-interaction. In Dagmar Barth-Weingarten, Elisabeth Reber \& Margret Selting (eds.), Prosody in interaction, 51-72. Amsterdam \& Philadelphia: John Benjamins.

Emma Rodero holds a doctorate and a Ph.D. in Communication from the University of Salamanca (Spain). She is Professor at the Universitat Pompeu Fabra (UPF, Barcelona), with a specialization in voice and prosody applied to radio and television. She is also Visiting Professor for numerous universities in Spain and in Latin America. She is author of Voice and Radio Presentation (IORTV), Radio Production (Cátedra), Advertising on Radio, (Ariel) and has published around forty journal articles and book chapters about radio communication. Address for correspondence: Departament of Communication, Pompeu Fabra University (UPF), Roc Boronat, 138, 08108, Barcelona, Spain 〈emma.rodero@upf.edu〉. 

Copyright of Text \& Talk is the property of De Gruyter and its content may not be copied or emailed to multiple sites or posted to a listserv without the copyright holder's express written permission. However, users may print, download, or email articles for individual use. 